\title{
3D Printing Geometric Scaffold Design Variation of Injectable Bone Substitutes (IBS) Paste
}

\author{
Dyah Hikmawati ${ }^{1, a)}$, Sarda Nugraheni ${ }^{1}$, Aminatun $^{1}$ \\ ${ }^{1}$ Department of Physics, Faculty of Science and Technology, Universitas Airlangga, Surabaya 60115, Indonesia \\ a) Corresponding author: dyah.hikmawati@yahoo.co.id or dyah-hikmawati@fst.unair.ac.id
}

\begin{abstract}
D printing technology application in tissue engineering could be provided by designing geometrical scaffold architecture which also functionates as drug delivery. For drug delivery scaffold on bone tuberculosis, the cell pore of the geometric design was filled with Injectable Bone Substitutes (IBS) which had streptomycin as anti-tuberculosis. In this study, scaffolds were synthesized in three cells geometric filled by Injectable Bone Substitutes (IBS), Hexahedron, Truccated Hexahedron, and Rhombicuboctahedron, which had $2.5 \mathrm{~mm} \times 2.5 \mathrm{~mm} \times 2.5 \mathrm{~mm}$ size dimension and $0.8 \mathrm{~mm}$ strut. The final design was printed in 3D with polylactic acid (PLA) filament using the FDM process (Fused Deposition Modelling). The composition of IBS paste was a mixture of hydroxyapatite (HA) and gelatine (GEL) 20\% w/v with a ratio of 60:40, streptomycin $10 \mathrm{wt} \%$ and hydroxypropyl methylcellulose (HPMC) 4\% w/v. It was then characterized using Fourier-transform infrared spectroscopy $(F T I R)$. Scaffold-paste characterization was included pore size test of 3D printing result before and after injected using Scanning Electron Microscope SEM, porosity test, and compressive strength test. The result showed that the pore of scaffold design was $1379 \mu \mathrm{m}$ and after injected with IBS paste, the pore leaving $231.04 \mu \mathrm{m}$ of size. The scaffold with IBS paste porosity test showed ranges between $40,78-70,04 \%$ while the compressive strength of before and after injected ranges between 1,110-634 MPa and 2,217-6,971 MPa respectively. From the test results, the scaffold 3D printing with IBS paste in this study had suitable physical characteristics to be applicated on cancellous bones which were infected by tuberculosis.
\end{abstract}

\section{INTRODUCTION}

Three dimensional (3D) printing, also known as Additive Layer Manufacturing, is a process to create objects in 3 dimensional or any other form from the digital model. 3D printing can be used widely to produce an object based on its complex geometries and interfaces design [1]. Scaffold design in tissue engineering is one of the applications of 3D printing in medicine to repair organ or tissue injury. In 2015, Jian An reported an experiment of 3D tissue and geometric scaffold design with 20 different polyhedral forms in unit cells [2]. Tan also fabricated the scaffold 3D printing in 2014, using alginate-based to construct tissue engineering [3]. In 2015, Chia upgraded the resolution of 3D printing using biomaterial polymer while Itoh Manabu was analyzing scaffold-free tubular tissue which created using Bio-3D Printer and proving cells remodelling and endothelialization when implanted in rat aortae [4-5]. An ideal 3D scaffold should have interconnected pores structure that can ease the cells proliferation, migration, and infiltration [6]. In 2016, Singh observed the function of scaffold on helping skin tissue regeneration and healing the injured areas [7].

Scaffold holds an essential role on keeping the shape of bone growth and also can be used as drug delivery by adding Injectable Bone Substitutes (IBS) paste and providing cells remodelling and regeneration materials on pores [8]. In 2014, Maulida synthesized IBS paste based on hydroxyapatite (HA), gelatine, and streptomycin for spinal tuberculosis. 3D printing innovation on scaffold printing and addition of IBS into scaffold pores facilitated the tuberculosis drug delivery process right on target and accelerated bone regeneration.

Fused Deposition Modelling (FDM) is one of a 3D printing technique by melting and extruding thermoplastic filament through the nozzle of layer by layer scaffold which is designed on printing area. At first, the scaffold was designed using Computer-Aided Design (CAD) software and then converted to .STL format with Polylactic Acid (PLA) filament. PLA is one of a plastic polymer which is made of biodegradable materials, such as corn, tapioca flour, or processed sugar cane. PLA filament was used on this scaffold printing study because its characteristics qualified the requirements of a scaffold; biodegradable, biocompatible, non-carcinogenic to local tissue, and also not impaired the tissue healing process. PLA is also environmentally friendly since it can be degraded naturally by light and bacteria hydrolysis so that it will be degradable in soil, recyclable, and renewable [9]. 
This study will discuss scaffold printing with polyhedral geometric design variation, Hexahedron, Truccated Hexahedron, and Rhombicuboctahedron, with $2.5 \mathrm{~mm}$ length of sides of unit cells and $0.8 \mathrm{~mm}$ strut using 3D printing method which then was added by IBS paste. The characterization included FTIR analysis to identify the functional groups of the IBS paste sample, and scaffold porosity test was calculated using Equation 1 as follows :

$$
\text { Porosity }=\frac{\mathrm{Ww}-W_{d}}{\mathrm{Ww}-W_{l}} x 100 \%
$$

Where :

$\mathrm{W}_{\mathrm{d}}=$ Initial Scaffold weight $(\mathrm{g})$

$\mathrm{W}_{\mathrm{l}}=$ Wet Scaffold weight $(\mathrm{g})$

$\mathrm{W}_{\mathrm{w}}=$ Dry Scaffold weight $(\mathrm{g})$.

Morphology test was carried out using the Scanning Electron Microscope (SEM) to identify the pore size of the scaffold sample before and after injected with IBS paste. Equation 2 was used to obtain the scaffold compressive strength as follows:

$$
\sigma=\frac{F}{A}
$$

Where $\sigma$ was compressive strength $(\mathrm{MPa}), \mathrm{F}(\mathrm{N})$ was the given force, while $\mathrm{A}\left(\mathrm{cm}^{2}\right)$ was the surface area of the scaffold sample.

$1 \mathrm{MPa}=1 \mathrm{~N} / \mathrm{mm}^{2}=10 \mathrm{~kg} / \mathrm{cm}^{2}$.

\section{EXPERIMENTAL METHOD}

Nano hydroxyapatite used in this study was obtained from Badan Tenaga Nuklir Nasional (BATAN) Jakarta, Indonesia originated from the fish scales. The gelatin was derived from cow skin purchased from 150 bloom Rousselot (Guangdong, China). The streptomycin sulfate (powder for injection) was obtained from PT. Meiji Indonesia. The hydroxypropyl methylcellulose (HPMC) was obtained from Sigma Aldrich H7509.

The injectable bone substitute (IBS) was synthesized by dissolving $20 \mathrm{w} / \mathrm{v} \%$ gelatin (GEL) in deionized water at a temperature of $40^{\circ} \mathrm{C}$ for one hour. The hydroxyapatite (HA) powder was added to that solution with the ration of HA and GEL 60:40, 10wt\% Streptomycin was added in the mixture. Meanwhile, HPMC 4\% w/v was dissolved in distilled water at $90^{\circ} \mathrm{C}$ then added to the solution of gelatin, hydroxyapatite and streptomycin at $40^{\circ} \mathrm{C}$ and stirred for six hours to produce a white IBS.

The tools used were freezer and lyophilizer, $10 \mathrm{cc}$ syringe, viscotester VT-04F RION, pH meter Benchtop OAKTON, and SEM FEI Inspect S50 Japan, while on designing the scaffold geometric 62-bit specification and 2 Gb RAM of Asus Laptop and AutoCAD 2012 program were used. The unit cells of the scaffold were first designed using "polyline" command and then directed to the Drawing Area to create a scaffold outline in AutoCAD. The finished scaffold was then reproduced using "copy" command on the Ribbon Bar, saved in .STL format, and printed in 3D using FDM technique.

The results of the unit cells scaffold design and 3D printing were then injected with IBS paste which made of hydroxyapatite, gelatine, HPMC, and streptomycin on the pore by using a syringe. The samples were then left for 24 hours to dry in room temperature.

\section{RESULTS AND DISCUSSIONS}

\section{Synthesized Result}

In this study, the scaffold pores variation were designed in Hexadron, Truccated Hexahedron, dan Rhombicuboctahedron. Each unit cells were designed in $2500 \mu \mathrm{m}$ size of length and strut of $800 \mu \mathrm{m}$ as many as 4 unit cells per 4 cells in unit pores using the AutoCAD. The designs were saved in .STL format and printed in 3D using PLA filament on FDM technique. The unit cells design, scaffold design, and 3D printing results were shown in Figure 1. The print results of scaffold samples were then injected with IBS paste based on hydroxyapatite (HA), gelatine, HPMC and streptomycin using a syringe and then left for 24 hours in room temperature. The final results of injected scaffolds were shown in Figure 2. 

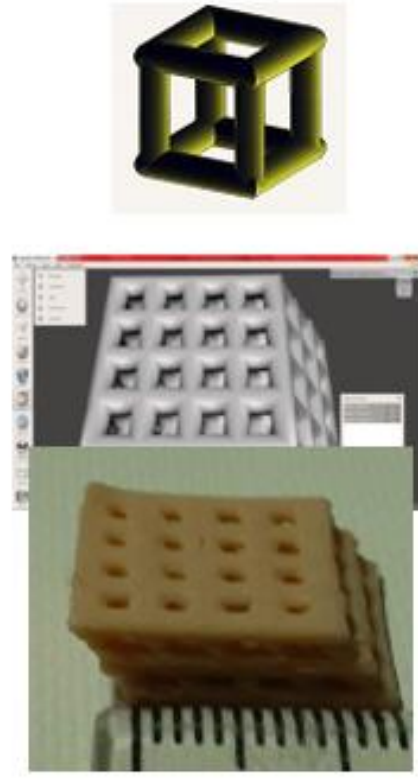

(a)
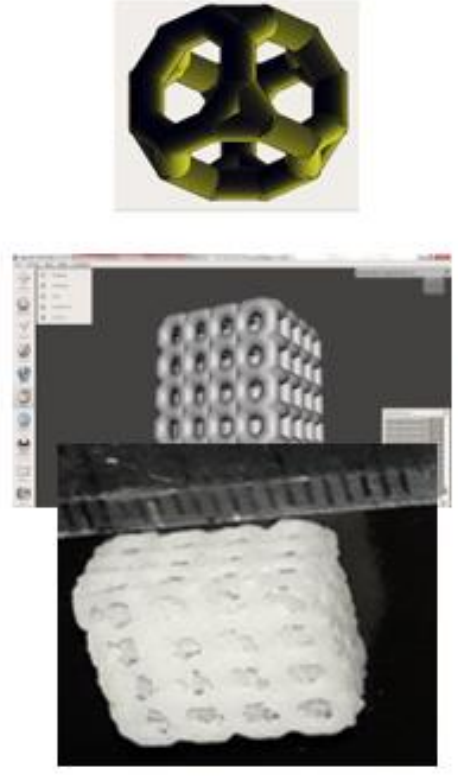

(b)
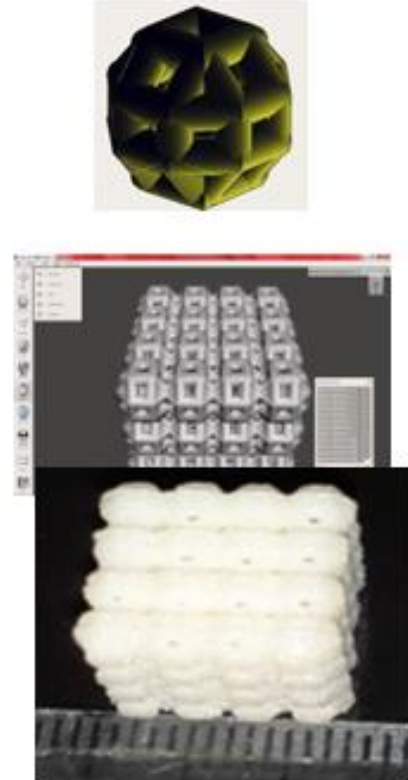

(c)

FIGURE 1. The unit cells design, scaffolds design, and 3D printings results :(a) Hexadron, (b) Truccated Hexahedron dan (c) Rhombicuboctahedron
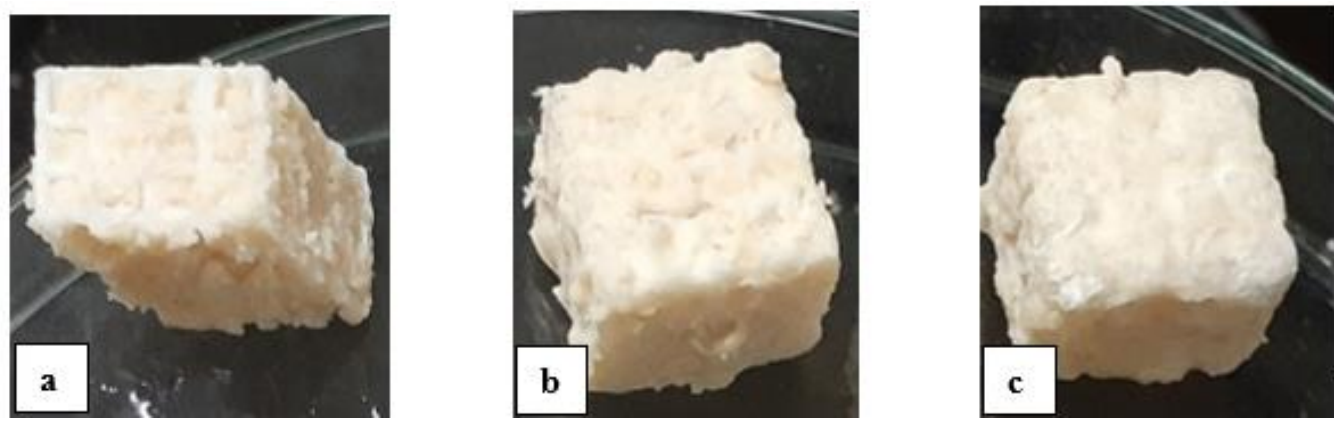

FIGURE 2. The final results of injected scaffolds: (a) Hexadron, (b) Truccated Hexahedron, (c) Rhombicuboctahedron

\section{Characterization Result}

The results of the scaffold with IBS paste were then characterized and analyzed using FTIR analysis, porosity test, SEM analysis, and compressive strength test.

\section{Fourier-Transform Infrared Spectroscopy (FTIR) Analysis Result.}

Infrared spectroscopy was carried out to identify the functional groups of the used synthesized material. The wavelength used on this FTIR analysis ranges between $4000-400 \mathrm{~cm}^{-1}$ for each IBS paste ingredients; hydroxyapatite (HA), gelatine, HPMC, streptomycin, and dried IBS paste sample using the freeze-drying method. Figure 3 presented the obtained spectrum. 


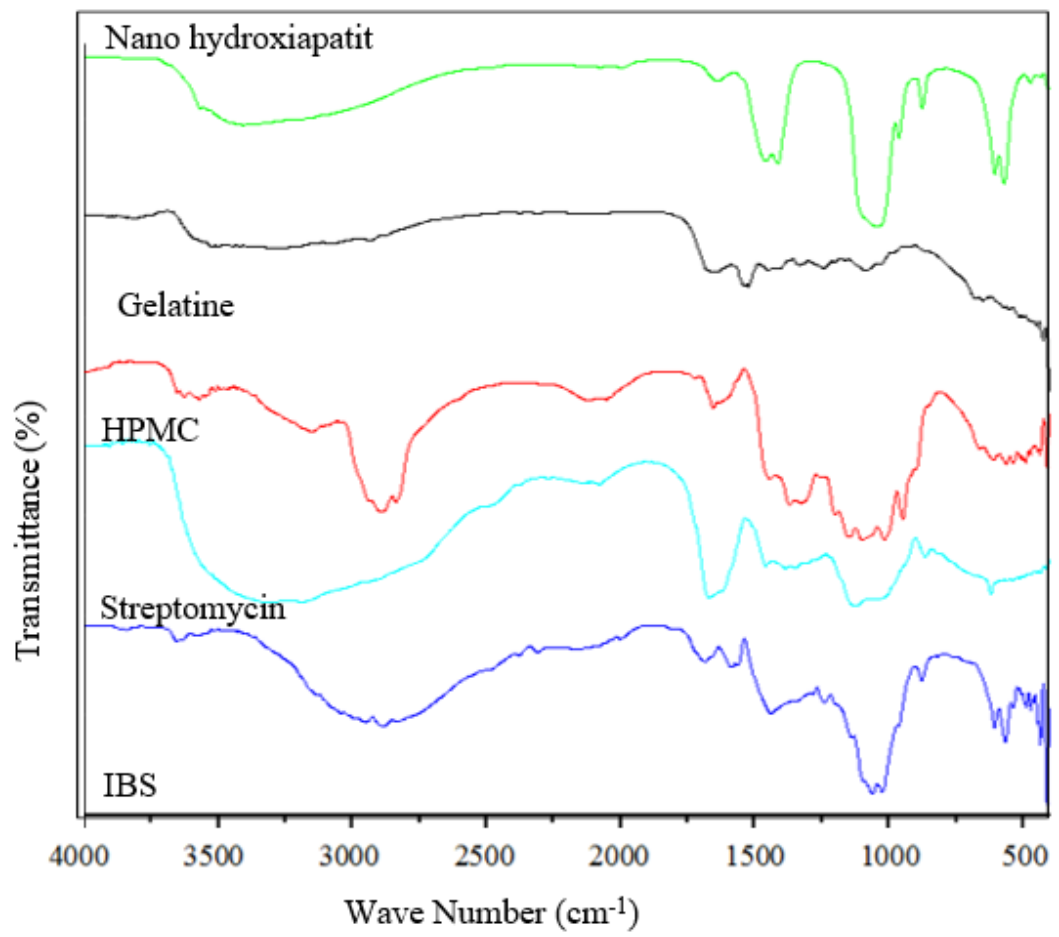

FIGURE 3. FTIR spectrum of IBS paste sample and its ingredients (hydroxyapatite, gelatine, HPMC, and streptomycin).

FTIR spectrum showed that the specific functional groups of dried IBS paste by the freeze-drying method could be identified as presented in Table 1.

TABLE 1.. Specific Functional Groups of IBS Paste

\begin{tabular}{lc}
\hline \multicolumn{1}{c}{ Identified Functional Groups } & $\begin{array}{c}\text { Wave Number } \\
\left(\mathbf{c m}^{\mathbf{- 1}}\right)\end{array}$ \\
\hline Stretching $\mathrm{OH}$ vibration & 3467.35 \\
\hline Stretching $\mathrm{C}-\mathrm{CH}_{3}$ vibration of HPMC & 2929.47 \\
\hline Carbonil (C=O) of gelatine & 1648.49 \\
\hline $\begin{array}{l}\mathrm{C}-\mathrm{NH} \text { bending of streptomycin from Amina functional } \\
\text { groups }\end{array}$ & 1578.22 \\
\hline
\end{tabular}

\section{Porosity Test Result}

Porosity is one of an essential parameter of bone tissue requirement since pores can provide sufficient space and nutrient diffusion for cells to grow. The percentage of porosity (\%porosity) on scaffold design and 3D printing paste result of Hexadron, Truccated Hexahedron dan Rhombicuboctahedron, were $62.31 \pm 0.002 \%$; $70.04 \pm 0.005 \%$; and $40 ; 78 \pm 0 ; 002 \%$ respectively. An ideal scaffold for bone cancellous should have $70 \%$ of \%porosity [11]. The biggest $\%$ porosity was shown by Truccated Hexahedron for $70.04 \pm 0.005 \%$, which was ideal for bone cancellous.

\section{Scanning Electron Microscopy (SEM) Analysis Result}

SEM analysis of scaffold characterization was carried out to obtain the diameter and morphology of the sample surface before and after injected with IBS paste. This study used SEM magnification of 50x to Truncated Hexahedron scaffold pore design, as this sample had the highest \%porosity. Figure 4a and 4b presented the result of SEM analysis. The strut structure of layer by layer pores barrier after PLA filament cumulation, and $1379 \mu \mathrm{m}$ pore size showed in Figure 4a. Meanwhile, Figure 4b presented the pore size of $231.04 \mu \mathrm{m}$ after injected with IBS paste. Smaller pore size showed that IBS paste was able to fill the scaffold pore and harden or setting. Based on the SEM analysis result, the Truncated Hexahedron of scaffold pore design qualified the pore bones size, range between $100-300 \mu \mathrm{m}$, so it was suitable for osteoblast growth and helped the formation of bone tissue [12]. 

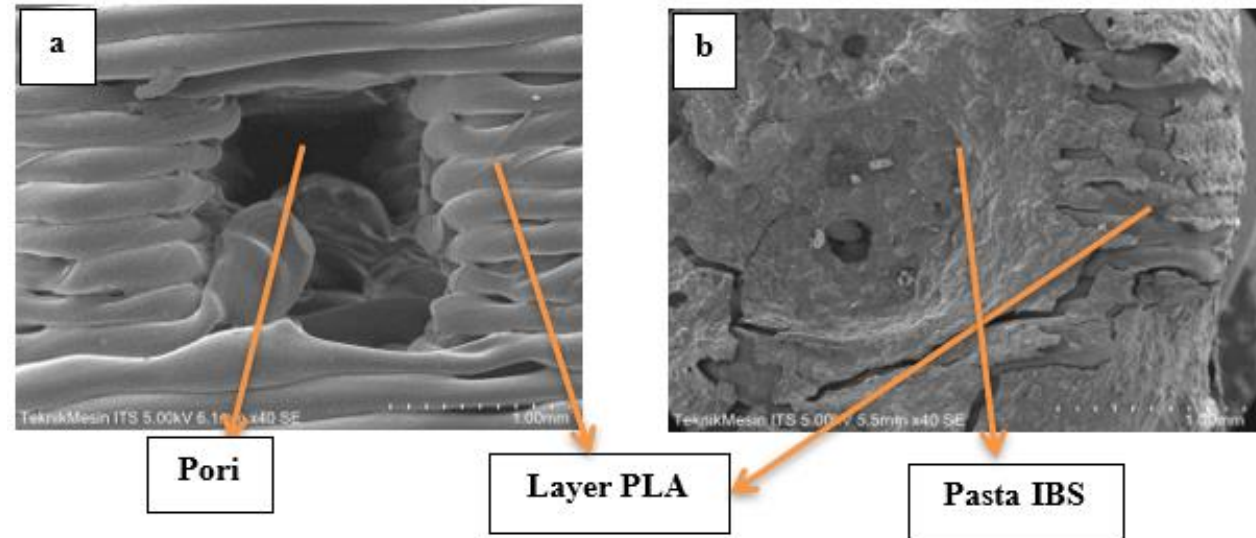

Figure 4. SEM analysis result of Truccated Hexahedron scaffold pore design: (a) before injected with IBS paste and (b) after injected with IBS paste.

TABLE 1. compressive strength test result of PLA scaffold before and after injected with IBS paste

\begin{tabular}{clcc}
\hline Unit Cells of Pore Design & $\begin{array}{c}\text { Compressive Strength Before } \\
\text { Injected with IBS Paste (Mpa) }\end{array}$ & $\begin{array}{c}\text { Compressive Strength After } \\
\text { Injected with IBS Paste (Mpa) }\end{array}$ \\
\hline A. & Hexadron & $1.11 \pm 0.089$ & $2.22 \pm 0.185$ \\
\hline B. & Truccated Hexahedron & $2.99 \pm 0.240$ & $5.42 \pm 0.452$ \\
\hline C. & Rhombicuboctahedron & $6.43 \pm 0.516$ & $6.97 \pm 0.581$ \\
\hline
\end{tabular}

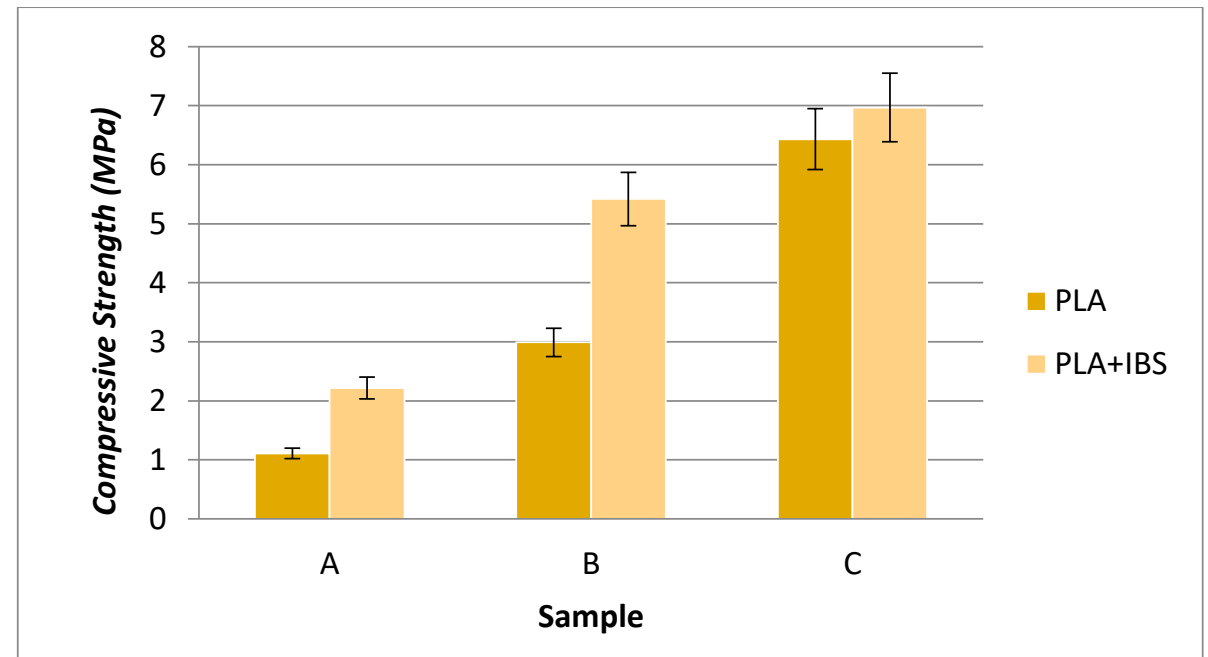

Figure 5. A compressive strength test result of the scaffold.

The addition of IBS paste into the scaffold increased the compressive strength of samples as presented in Figure 5. The lowest increment of compressive strength was on Rhombicuboctahedron unit cell scaffold design since it had a smaller pore than Truccated Hexahedron so the amount of injected IBS paste also lesser. The compressive strength of cancellous bone substitute was between 2-12 MPa and between 1.5-7.8 MPa for vertebrae [13]. From the compressive strength test result, all of the variations of scaffold design qualified for cancellous vertebrae bone scaffold.

\section{CONCLUSION}

3. The 3D printing result of geometric design scaffold indicated layer by layer of PLA as the scaffold partition. The pore of Truccated Hexahedron scaffold design was $1379 \mu \mathrm{m}$ of size and filled by IBS paste after injected. FTIR analysis result on IBS paste showed that no new functional groups formed.

4. The porosities of scaffolds with IBS paste were between 40,78-70,04\%, and Truccated Hexahedron had the biggest pore design. From SEM analysis, Truccated Hexahedron left a pore size for $231.04 \mu \mathrm{m}$ after injected with IBS paste. 
The compressive strength of this scaffold variation was 1.110-6.434 MPa and increased for 2.217-6.971 MPa after injected with IBS paste.

\section{REFERENCE}

1. Al'Aref, J Subhi. 2018. 3D Printing Applications in Cardiovascular Medicine. London : Elsevier Inc.

2. Jia An, Joanne Ee Mei Teoh, Chee Kai Chua. 2015. Design and 3D Printing of Scaffolds and Tissues Engineering.

3. Tan, Yu.. 2014. 3D Printing Facilitated Scaffold-free Tissue Unit Fabrication.1:1-16.

4. Chia Helena N dan Wu Benjamin M.. 2015. Recent advantaces in 3D printing of Biomaterials.9:1-4.

5. Itoh M, Nakayama K, Nokuguchi R and Furukawa K. 2015. Scaffold-Free Tubular Tissue Created by a Bio$3 D$ Printer Undergo Remodelling and Endothelialization when Implanted in Rat Aortae

6. Q.L.Loh, C. Choong, Three-dimensional Scaffold for Tissues Engineering Applications: Role of porosity and pore size. Tissues Engineering Part B Review, 2013, 19(6)485-502.

7. Singh Deepti dan Singh Dolly. 2016. 3D Printing of Scaffold for Cells Delivery Advences in Skin Tissue Engineering. 8:1-19.

8. H. N.Maulida, D. Hikmawati, and A. S. Budiatin, "Injectable Bone Substitute Paste Based on Hydroxyapatite, Gelatin and Streptomycin for Spinal Tuberculosis,” J. Spine, 4, pp. 4-7, 2015.

9. Ren, Jie. 2010. Synthesis, Modification, Processing and Applications. Shanghai: Tsinghua University Press.

10. Kokubo T dan Takadama H.. 2006. How Useful is SBF in Predicting in Vivo Bone Bioactivity. 15:2907-15

11. Barralet JE, Gaunt T, Wright AJ, Gibson IR, Knowles JC. 2002. Effect of Porosity Reduction by Compaction on Compressive Strength and Microstucture of Calcium Phosphate Cement. 63:1-9.

12. Ficai, A., Andrnescu, E., Voicu, G., Ficai, D. 2011. Advances in Collagen/Hidroxyapatite Composite Material. In Tech.

13. Mosekilde, Lis. And Mosekilde, Leif. 1989. Sex Different in age-related changes in vertebral body size, density and biomechanical competence in normal individuals. 11:67-73. 